\author{
United Nations Educational Scientific and Cultural Organization \\ and \\ International Atomic Energy Agency
}

THE ABDUS SALAM INTERNATIONAL CENTRE FOR THEORETICAL PHYSICS

\title{
LOCAL ENERGY EQUATION FOR TWO-ELECTRON ATOMS AND RELATION BETWEEN KINETIC ENERGY AND ELECTRON DENSITIES
}

\author{
N.H. March \\ Department of Physics, University of Antwerp (RUCA), \\ Groenenborgerlaan 171, B-2020 Antwerp, Belgium, \\ Oxford University, Oxford, England \\ and \\ The Abdus Salam International Centre for Theoretical Physics, Trieste, Italy.
}

\begin{abstract}
In early work, Dawson and March [J. Chem. Phys. 81, 5850 (1984)] proposed a local energy method for treating both Hartree-Fock and correlated electron theory. Here, an exactly solvable model two-electron atom with pure harmonic interactions is treated in its ground state in the above context. A functional relation between the kinetic energy density $t(r)$ at the origin $r=0$ and the electron density $\rho(r)$ at the same point then emerges. The same approach is applied to the Hookean atom, in which the two electrons repel with Coulombic energy $e^{2} / r_{12}$, with $r_{12}$ the interelectronic separation, but are still harmonically confined. Again the kinetic energy density $t(r)$ is the focal point, but now generalization away from $r=0$ is also effected. Finally, brief comments are added about $\mathrm{He}$-like atomic ions in the limit of large atomic number.
\end{abstract}

MIRAMARE - TRIESTE

August 2002 


\section{Background and outline}

In an early study, Dawson and March ${ }^{1}$ set out the so-called local energy method for treating atomic and molecular systems both at Hartree-Fock (HF) and correlated levels. The present investigation is related in that a local energy equation is first set up for treating two-electron atoms in which both 'electron-nuclear' and 'electron-electron' interactions are left to be defined.

Suppose then the 'electron-nuclear' potential energy is denoted by $U(r)$ and the 'electronelectron' interaction energy by $W\left(r_{12}\right)$, with $r_{12}$ the interelectronic separation of the (assumed) spin-compensated ground state of the model atom thereby defined.

Then, if $\Psi\left(\mathbf{r}_{1}, \mathbf{r}_{2}\right)$ and $E$ are, respectively, the spatial (symmetric) ground-state wave function and corresponding eigenvalue, the most general local energy equation reads

$$
E=\frac{H \Psi}{\Psi}
$$

where $H$ denotes the Hamiltonian operator given explicitly by

$$
H=-\frac{1}{2} \nabla_{1}^{2}-\frac{1}{2} \nabla_{2}^{2}+U\left(r_{1}\right)+U\left(r_{2}\right)+W\left(r_{12}\right)
$$

While the above local energy method applies at each pair of vectors $\left(\mathbf{r}_{1}, \mathbf{r}_{2}\right)$, we shall now choose to integrate out information involving $\mathbf{r}_{2}$. Thus, for these two-electron atoms the ground-state electron density $\rho\left(r_{1}\right)$ is given by

$$
\rho\left(r_{1}\right)=2 \int \Psi^{2}\left(\mathbf{r}_{1}, \mathbf{r}_{2}\right) d \mathbf{r}_{2}
$$

since the normalized ground-state wave function $\Psi$ is real. Multiplying eqn.(1.1) by $\Psi^{2}$ and integrating over all $\mathbf{r}_{2}$ yields

$$
\begin{aligned}
\frac{E \rho\left(r_{1}\right)}{2}= & t\left(r_{1}\right)+\frac{U\left(r_{1}\right) \rho\left(r_{1}\right)}{2}+\int U\left(r_{2}\right) \Psi^{2}\left(\mathbf{r}_{1}, \mathbf{r}_{2}\right) d \mathbf{r}_{2} \\
& +\int W\left(r_{12}\right) \Psi^{2}\left(\mathbf{r}_{1}, \mathbf{r}_{2}\right) d \mathbf{r}_{2} .
\end{aligned}
$$

It is clear that the first term on the right-hand side of eqn.(1.4) is the kinetic energy density, denoted by $t\left(r_{1}\right)$, which is precisely defined from the wave function form $\Psi \nabla^{2} \Psi$, and not from the positive-definite $(\nabla \Psi)^{2}$ alternative. However, as will be invoked below, these definitions differ only by a multiple of the Laplacian of the electron density, namely $\nabla^{2} \rho$.

From eqn.(1.4), the local energy equation at the heart of the present study of two-electron atoms reads

$$
\begin{aligned}
E= & \frac{2 t\left(r_{1}\right)}{\rho\left(r_{1}\right)}+U\left(r_{1}\right)+\frac{2}{\rho\left(r_{1}\right)} \int U\left(r_{2}\right) \Psi^{2}\left(\mathbf{r}_{1}, \mathbf{r}_{2}\right) d \mathbf{r}_{2} \\
& +\frac{2}{\rho\left(r_{1}\right)} \int W\left(r_{12}\right) \Psi^{2}\left(\mathbf{r}_{1}, \mathbf{r}_{2}\right) d \mathbf{r}_{2} .
\end{aligned}
$$

The outline of the rest of this paper is then as follows. In section 2 immediately below eqn.(1.5) is applied to a model atom in which both $U(r)$ and $W\left(r_{12}\right)$ are harmonic in form, such 
a proposal going back at least to Moshinsky ${ }^{2,3}$. The main result we focus on relates to the limit $r_{1} \rightarrow 0$ of the ratio of $t\left(r_{1}\right)$ to $\rho\left(r_{1}\right)$. Section 3 utilizes eqn.(1.5) for the so-called Hookean atom, going back to Kestner and Sinanoshu ${ }^{4-6}$. Here, harmonic springs still tie electrons 1 and 2 to the 'nucleus' at the origin $r=0$, but the interaction potential energy $W\left(r_{12}\right)$ is now the Coulombic repulsion $e^{2} / r_{12}$. Again, the kinetic energy density $t(r)$ is the main focus of attention. Section 4 treats then, but now quite briefly, $\mathrm{He}$-like atomic ions with large atomic number $Z$ although, as throughout the paper, non-relativistic quantum mechanics is retained. Section 5 constitutes a summary, plus some sugestions for future studies.

\section{Two-electron atom with purely harmonic interactions}

For the atom proposed by Moshinsky ${ }^{2,3}$, the choices of 'external' potential $U(r)$ and interaction term $W\left(r_{12}\right)$ are as follows:

$$
U(r)=\frac{1}{2} r^{2} ; \quad W\left(r_{12}\right)=\frac{1}{2} K r_{12}^{2},
$$

where $K$ evidently measures the relative strength of $U$ ad $W$. The spatial form of the groundstate wave function is known to be

$$
\begin{aligned}
\Psi\left(\mathbf{r}_{1}, \mathbf{r}_{2}\right)= & \pi^{-3 / 2}(1+2 K)^{3 / 8} \exp \left(-\frac{r_{1}^{2}+r_{2}^{2}}{2}\right) \\
& \times \exp \left(-\frac{1}{2}\{\alpha-1\}\left|\mathbf{r}_{1}-\mathbf{r}_{2}\right|^{2}\right)
\end{aligned}
$$

the quantity $\alpha$, which measures the ground-state energy $E$ through

$$
E=3 \alpha
$$

being defined as a function of $K$ by

$$
\alpha=\frac{1}{2}\left[(1+2 K)^{1 / 2}+1\right] .
$$

In principle, one can insert directly eqns.(2.1) - (2.4) into the local energy eqn.(1.4). However, we shall restrict ourselves in the present section to taking the limit of the r.h.s. of eqn.(1.4) as $r_{1} \rightarrow 0$, and shall thereby focus all attention on the calculation of the limit

$$
\lim _{r_{1} \rightarrow 0} \frac{t\left(r_{1}\right)}{\rho\left(r_{1}\right)} \equiv \frac{t(0)}{\rho(0)} .
$$

Since $U\left(r_{1}\right)$ from eqn.(2.1) tends to zero as $r_{1} \rightarrow 0$, we readily obtain

$$
\begin{aligned}
\frac{(t(0)}{\rho(0)}=\frac{E}{2} & -\frac{1}{\rho(0)} \int \frac{1}{2} r_{2}^{2} \Psi^{2}\left(0, \mathbf{r}_{2}\right) d \mathbf{r}_{2} \\
& -\frac{1}{\rho(0)} \int \frac{1}{2} K r_{2}^{2} \Psi^{2}\left(0, \mathbf{r}_{2}\right) d \mathbf{r}_{2} .
\end{aligned}
$$


Evidently, the calculation of the ratio of kinetic energy density $t$ to electron density $\rho$ at the origin $r=0$ reduces to the evaluation of the integral $I$ defined by

$$
I=\int r_{2}^{2} \Psi^{2}\left(0, r_{2}\right) d \mathbf{r}_{2}
$$

From eqn.(2.2),

$$
\Psi^{2}\left(0, r_{2}\right)=n^{2} \exp \left(-\alpha r_{2}^{2}\right):
$$

where $n^{2}=\pi^{-3}(1+2 K)^{3 / 4}$.

Thus, by simple quadrature one has the explicit result

$$
\frac{I}{4 \pi n^{2}}=\int_{0}^{\infty} r^{4} \exp \left(-\alpha r^{2}\right) d r=\frac{3}{8} \frac{\pi^{1 / 2}}{\alpha^{5 / 2}} .
$$

Using next a result of the study of March et al. ${ }^{7}$, one may write the normalization factor $n$ in terms of the electron density $\rho(0)$ at the origin $r=0$ as

$$
n^{2}=\left[\frac{\rho(0)}{2}\right]\left[\frac{\alpha}{\pi}\right]^{3 / 2}
$$

Hence by substituting eqn.(2.10) into eqn.(2.9) we readily find

$$
I=\frac{3}{4} \frac{\rho(0)}{\alpha} .
$$

Returning to the local energy equation in the form (2.6), and utilizing eqns.(2.7) and eqn.(2.11), one finds the ratio $t(0) / \rho(0)$ to be given by

$$
\frac{t(0)}{\rho(0)}=\frac{3 \alpha}{2}-\frac{3}{8 \alpha}(1+K) \text {. }
$$

The final step in obtaining a physically significant relation between $t(0)$ and $\rho(0)$ in this model two-electron atom is to express the r.h.s. of eqn.(2.12) solely in terms of $\rho(0)$ itself.

First of all, from eqn.(2.4) it follows readily that

$$
K=2 \alpha^{2}-2 \alpha
$$

and using eqn.(2.13) in eqn.(2.12) then yields the result

$$
\frac{t(0)}{\rho(0)}=\frac{3}{8}\left[2 \alpha+2-\frac{1}{\alpha}\right]
$$

But from eqn.(10) of March et al. ${ }^{7}$, one can write

$$
\frac{1}{\alpha}=2-\pi\left\{\frac{\rho(0)}{2}\right\}^{2 / 3} .
$$

Using eqn.(2.15) in eqn.(2.14), it is evident that $t(0) / \rho(0)$ is a simple function of $\rho(0)^{2 / 3}$, namely

$$
\frac{t(0)}{\rho(0)}=\frac{3}{8} \frac{\left[2+2 \pi\left\{\frac{\rho(0)}{2}\right\}^{2 / 3}-\pi^{2}\left\{\frac{\rho(0)}{2}\right\}^{4 / 3}\right]}{\left[2-\pi\left\{\frac{\rho(0)}{2}\right\}^{2 / 3}\right]} .
$$

Since one of the current aims of density functional theory is to obtain the kinetic energy density $t$ as a functional of the ground-state density $\rho$, eqn.(2.16) for the interacting two-electron 'harmonic' atom is a significant result in that context. 


\section{Local energy equation plus kinetic energy density for two- electron Hookean atom}

Let us turn to the second of the model atoms already referred to in section 1, namely the twoelectron Hookean atom. While characterized in general by the choices of external potential $U(r)$ and electron-electron interaction $W\left(r_{12}\right)$ :

$$
U(r)=\frac{1}{2} k r^{2} ; \quad W\left(r_{12}\right)=e^{2} / r_{12},
$$

we shall content ourselves with the solvable case ${ }^{5,6}$ when $k=1 / 4$. The important point to be stressed here is the retention of the Coulombic repulsion energy $e^{2} / r_{12}$ in this model atom, which then subsumes the key electron-electron cusp condition.

For the above choice of force constant $k=1 / 4$, the spatial ground-state wave function is known to be 5,6

$$
\Psi\left(\mathbf{r}_{1}, \mathbf{r}_{2}\right)=n \exp \left(-\frac{\left|\mathbf{r}_{1}+\mathbf{r}_{2}\right|^{2}}{8}\right) \exp \left(-\left|\mathbf{r}_{2}-\mathbf{r}_{1}\right|^{2} / 8\right)\left(1+\frac{\left|\mathbf{r}_{2}-\mathbf{r}_{1}\right|}{2}\right),
$$

the normalization factor $n$ having the value

$$
n=1 /\left[2 \pi^{5 / 4}\left(5 \pi^{1 / 2}+8\right)^{1 / 2}\right] .
$$

The ground-state electron density in this model with $k=1 / 4$ is given by ${ }^{6}$

$$
\begin{gathered}
\rho(r)=\frac{\pi(2 \pi)^{1 / 2} n^{2}}{r} \exp \left(-\frac{r^{2}}{2}\right)\left\{7 r+r^{3}+\left(8 /(2 \pi)^{1 / 2}\right) r \exp \left(-\frac{r^{2}}{2}\right)\right. \\
\left.+4\left(1+r^{2}\right) \operatorname{erf}\left(r / 2^{1 / 2}\right)\right\}
\end{gathered}
$$

where the error function is defined as

$$
\operatorname{erf}(x)=\frac{2}{\pi^{1 / 2}} \int_{0}^{x} \exp \left(-y^{2}\right) d y
$$

Following section 2, we shall again focus on the consequences of the local energy eqn.(1.4) at the origin $r=0$. Then we may write, using $k=1 / 4$ in eqn.(3.1),

$$
E=\frac{2 t(0)}{\rho(0)}+\frac{2}{\rho(0)} \int \frac{1}{8} r_{2}^{2} \Psi^{2}\left(0, r_{2}\right) d \mathbf{r}_{2}+\frac{2}{\rho(0)} \int \frac{e^{2}}{r_{2}} \cdot \Psi^{2}\left(0, r_{2}\right) d \mathbf{r}_{2}
$$

But from eqn.(3.2)

$$
\Psi^{2}\left(0, r_{2}\right)=n^{2} \exp \left(-\frac{1}{2} r_{2}^{2}\right)\left(1+\frac{r_{2}}{2}\right)^{2}
$$

and for the density $\rho(0)$ at the origin one finds from eqn.(3.4) the explicit form

$$
\rho(0)=n^{2}\left[7 \cdot 2^{1 / 2} \pi^{3 / 2}+16 \pi\right]
$$

which can be viewed as the analogue of eqn.(2.15) obtained earlier for the Moshinsky atom. 


\subsection{Kinetic energy density related to electron density for all values of $r$}

Instead of focussing solely on $t(0)$ appearing in the local energy form (3.6), it will be instructive below to study $t(r)$ for all $r$. While this is directly accessible from the local energy eqn.(1.4) by inserting eqns.(3.1) and (3.2), one can also obtain it via the kinetic energy density tensor $t_{\alpha \beta}(r)$ defined by Holas and March ${ }^{8}$, though its trace now corresponds to the positive-definite form of kinetic energy density proportional to $(\operatorname{grad} \psi)^{2}$ in wave function terms: we shall denote this form by $t_{\text {grad }}(r) \equiv t_{g}(r)$. The explicit relation between $t(r)$ appearing in the local energy eqn.(1.4) and $t_{g}(r)$ is

$$
t(r)=t_{g}(r)-\frac{1}{4} \nabla^{2} \rho(r) .
$$

For a convenient form of $t_{\alpha \beta}(r)$ for present purposes, we next appeal to the work of Qian and Sahni ${ }^{9}$, and in particular to their eqn.(A18) to write

$$
t_{g}(r)=f(r)+3 k(r)
$$

where in ref.10 explicit expressions are recorded in eqns.(A19) and (A20) for $f$ and $k$ respectively. Forming the combination $f+3 k$ appearing in eqn.(3.10) leads to the important simplification that Dawson's integral appearing in the separate expressions for $f$ and $k$ cancels in $f+3 k$, and only $\operatorname{erf}\left(r / 2^{1 / 2}\right)$ appearing in $\rho(r)$ given in eqn.(3.4) above remains, apart from simple explicit functions. We then obtain $t_{g}(r)$ from eqn.(3.10) as

$$
\begin{aligned}
t_{g}(r)= & \frac{r^{2} \rho(r)}{8}-\frac{\pi^{3 / 2}}{2^{1 / 2}} n^{2} r^{2} \exp \left(-\frac{r^{2}}{2}\right)+2^{1 / 2} \pi^{3 / 2} n^{2} \frac{\left(1-r^{2}\right)}{r} \operatorname{erf}\left(r / 2^{1 / 2}\right) \\
& -2 \pi n^{2} \exp \left(-r^{2}\right)+\frac{\pi^{3 / 2}}{2^{1 / 2}} n^{2} \exp \left(-\frac{r^{2}}{2}\right)
\end{aligned}
$$

It is now a fairly straightforward matter to take the limit of $t_{g}(r)$ as $r \rightarrow 0$, the definition (3.5) being invoked in the process, as well as the value of $n^{2}$ given in eqn.(3.3). The desired result is then found to be

$$
t_{g}(0)=\frac{\pi^{3 / 2} n^{2}}{2^{1 / 2}}=\frac{1}{2^{5 / 2} \pi\left[5 \pi^{1 / 2}+8\right]} .
$$

Finally, we invoke eqn.(3.9) to obtain $t(0)$ from $t_{g}(0)$, via the Laplacian of the density $\rho(r)$, the 'correction' to $t_{g}(0)$ being

$$
-\left.\frac{1}{4} \nabla^{2} \rho\right|_{r=0}=\frac{\left[\frac{15}{16}\left(\frac{\pi}{2}\right)^{1 / 2}+1\right] 2 \pi^{3 / 2}}{\left[5 \pi^{1 / 2}+8\right]} .
$$

Then the kinetic energy density $t(r)$ at the origin $r=0$ has the value

$$
t(0)=\frac{1}{2^{5 / 2} \pi\left[5 \pi^{1 / 2}+8\right]}\left[1+2^{7 / 2} \pi^{5 / 2}\left\{1+\frac{15}{16}\left(\frac{\pi}{2}\right)^{1 / 2}\right\}\right]
$$

Thus, from eqns.(3.3), (3.8), (3.12), and (3.14) we obtain the analogue of eqn.(2.12) derived earlier for the Moshinsky atom, when eqn.(3.8) is used directly for $\rho(0)$. 
Returning to the basic $r$-dependent eqn.(3.11), let us multiply throughout by $r$ to find

$$
\begin{gathered}
r t_{g}(r)=\frac{r^{3} \rho(r)}{8}-\frac{\pi^{3 / 2}}{2^{1 / 2}} n^{2} r^{3} \exp \left(-r^{2} / 2\right) \\
+2^{1 / 2} \pi^{3 / 2} n^{2}\left(1-r^{2}\right) \operatorname{erf}\left(r / 2^{1 / 2}\right)-2 \pi n^{2} r \exp \left(-r^{2}\right) \\
+\frac{\pi^{3 / 2}}{2^{1 / 2}} n^{2} r \exp \left(-\frac{r^{2}}{2}\right)
\end{gathered}
$$

It turns out that, to eliminate the error function, one needs to differentiate eqn.(3.15) three times, the result being set out in eqn.(A6) of the Appendix. The desired result relating $t_{g}(r)$ and $\rho(r)$ is then of the form

$$
\left(r^{4}-r^{2}\right) t_{g}^{\prime \prime \prime}+3\left(r^{3}-r\right) t_{g}^{\prime \prime}=R_{0}(r) \rho+R_{1}(r) \rho^{\prime}+R_{2}(r) \rho^{\prime \prime}+R_{3}(r) \rho^{\prime \prime \prime}
$$

Here the $R_{i}(r)$ denote polynomials in $r$, which are recorded explicitly in the Appendix. Eqn.(3.16) represents the functional relation between the positive definite kinetic energy density $t_{g}(r)$ and the electron density $\rho(r)$ plus its low-order derivatives. It completes the sought-after density functional result for the two-electron Hookean atom. One boundary condition which $t_{g}(r)$ must satisfy on integration of eqn.(3.16) is the form (3.12) for $t_{g}(0)$.

\section{$4 H e$-like atomic ions for large atomic number $Z$}

Let us first neglect the electron-electron interaction term $e^{2} / r_{12}$ relative to the electron-nuclear coupling $-Z e^{2} / r$, an approximation which becomes increasingly valid for large $Z$. Then with $U(r)=-Z e^{2} / r$, one can derive a differential equation for the kinetic energy density $t(r)$ in terms of $U$ and the electron density $\rho(r)$ as ${ }^{11}$

$$
t^{\prime}(r)=-\frac{3}{2} U^{\prime}(r) \rho-\frac{\rho^{\prime}}{4 r^{2}}-\frac{\rho^{\prime \prime}}{2 r}-\frac{\rho^{\prime \prime \prime}}{8} .
$$

Let us form $t_{g}(r)$ using eqn.(3.9) as

$$
t_{g}^{\prime}=-\frac{3}{2} U^{\prime}(r) \rho-\frac{3 \rho^{\prime}}{4 r^{2}}+\frac{\rho^{\prime \prime \prime}}{8} .
$$

But $U^{\prime}(r)=Z e^{2} / r^{2}$ in this case, and therefore multiplying (4.2) by $\frac{r^{2}}{\rho}$ yields

$$
\frac{r^{2} t_{g}^{\prime}}{\rho}=-\frac{3}{2} Z e^{2}-\frac{3 \rho^{\prime}}{4 \rho}+\frac{r^{2} \rho^{\prime \prime \prime}}{8 \rho} .
$$

Differentiating with respect to $r$ given the differential equation relating $t_{g}$ and $\rho$ in the large $Z$ limit of non-relativistic quantum mechanics as

$$
r^{2} \rho t_{g}^{\prime \prime}+\left[2 r \rho-r^{2} \rho^{\prime}\right] t_{g}^{\prime}=\frac{3}{4} \rho^{\prime 2}-\frac{3}{4} \rho \rho^{\prime \prime}+\frac{1}{4} r \rho \rho^{\prime \prime \prime}+\frac{r^{2} \rho \rho^{I V}}{8}-\frac{r^{2}}{8} \rho^{\prime} \rho^{\prime \prime \prime} .
$$

Again, but now in the limit $Z \rightarrow \infty$ for $H e$-like atomic ions, one has a functional relationship between the kinetic energy density $t_{g}(r)$ and the electron density $\rho(r)$; the counterpart of the 
exact eqn.(3.16) for the Hookean atom. It would seem interesting for the future to make use of the Schwartz ${ }^{12}$ density $\rho(r)$ which subsumes $e^{2} / r_{12}$ in lowest order. But presently, there is no route of equivalent reliability for the calculation of $t_{g}(r)$ with inclusion of $e^{2} / r_{12}$, and so we shall not pursue the matter further here.

We note finally as an alternative to eqn.(4.4), that for the non-interacting case when $e^{2} / r_{12}$ is omitted the positive definite kinetic energy $t_{g}(r)$ is explicitly given by the von Weizsäcker form 13

$$
t_{g}(r)=\frac{1}{8}{\frac{\rho^{\prime}}{\rho(r)}}^{2}
$$

and hence

$$
t_{g}^{\prime}(r)=\frac{1}{4} \frac{\rho^{\prime} \rho^{\prime \prime}}{\rho}-\frac{1}{8} \frac{\rho^{\prime 3}}{\rho^{2}} .
$$

Multiplying (4.6) through by $\rho^{2}$ and differentiating the result with respect to $r$ readily yields, after multiplying finally by $r^{2}$ :

$$
r^{2} \rho^{2} t_{g}^{\prime \prime}+2 r^{2} \rho \rho^{\prime} t_{g}^{\prime}=\frac{r^{2}}{4} \rho \rho^{\prime \prime 2}-\frac{r^{2}}{8} \rho^{\prime 2} \rho^{\prime \prime}+r^{2} \frac{\rho \rho^{\prime} \rho^{\prime \prime \prime}}{4} .
$$

Subtracting eqn.(4.7) from eqn.(4.4) times $\rho(r)$ then gives

$$
\begin{aligned}
{\left[2 r \rho^{2}-3 r^{2} \rho \rho^{\prime}\right] t_{g}^{\prime}=} & \frac{3}{4} \rho \rho^{\prime 2}-\frac{3}{4} \rho^{2} \rho^{\prime \prime}+\frac{1}{4} r \rho^{2} \rho^{\prime \prime \prime}+\frac{r^{2} \rho^{2} \rho^{I V}}{8} \\
& -\frac{r^{2}}{4} \rho \rho^{\prime \prime} 2+\frac{r^{2}}{8} \rho^{\prime 2} \rho^{\prime \prime}-\frac{3}{8} r^{2} \rho \rho^{\prime} \rho^{\prime \prime \prime}
\end{aligned}
$$

which is a functional relationship between $t_{g}^{\prime}$ and electron density $\rho$ plus its derivatives up to, and including, $\rho^{I V}$. From Kato's theorem ${ }^{14}$, we have that

$$
\left.\frac{\partial \rho}{\partial r}\right|_{r=0}=-\frac{2 Z}{a_{0}} \rho(r=0)
$$

and hence, by combining eqns.(4.5) and (4.9):

$$
t_{g}(r=0)=\frac{1}{2} \frac{Z^{2}}{a_{0}^{2}} \rho(r=0) .
$$

Thus $t_{g}(r=0)$ is fixed from knowledge of $\rho(r=0)$, plus however the value of the atomic number $Z$.

\section{Summary and future directions}

The main results of the present study of two-electron atoms are:

(i) The local energy eqn.(1.4) for general 'external' potential $U(r)$ and 'electron-electron' interaction $W\left(r_{12}\right)$.

(ii) The density functional relationship (2.16) between the kinetic energy density $t(0)$ and $\rho(0)$, for the Moshinsky atom for which both $U$ and $W$ are of harmonic form. 
(iii) The functional relationship between the positive definite kinetic energy density $t_{g}(r)$ and the electron density $\rho(r)$ embodied in the exact differential eqn.(3.16) for the Hookean atom, for which $U$ is still harmonic, but $W\left(r_{12}\right)=e^{2} / r_{12}$, the electron-electron cusp condition thereby being subsumed completely.

(iv) For helium-like atomic ions in the limit of sufficiently large atomic number $Z$ that $e^{2} / r_{12}$ can be neglected, the differential eqn.(4.4), or its alternative form (4.8), relating $t_{g}(r)$ to $\rho(r)$.

For the future, it would seem to be of some interest to attempt the generalization of eqns.(4.4) and/or (4.8) to include the electron-electron interaction to lowest order, for large atomic number $Z$. Further results for the Moshinsky atom which would be worthwhile in the future involve the generalization of eqn.(2.16) away from $r=0$, and in particular the connected study of nonlocality in relating $t(r)$ and $\rho(r)$ which is not apparent from this equation.

\section{Acknowledgments}

It is a pleasure to acknowledge numerous valuable discussions with Drs. C. Amovilli, F. Bartha, F. Bogar and T. Gál and Professors I.A. Howard, Á. Nagy and P. Senet on the general area embraced by the present study. Finally, thanks are due to Professor Yu Lu for making possible the author's visit to the Abdus Salam International Centre for Theoretical Physics, Trieste, Italy, in 2002, and for generous hospitality during the period in which the work was carried out. 


\section{Appendix A}

Some details on Hookean atom including explicit forms of polynomials $R_{i}(r)$ in differential eqn.(3.16) for kinetic energy density $t_{g}(r)$.

The detailed way in which one passes from eqn.(3.15) to the final differential eqn.(3.16) giving the kinetic energy density $t_{g}(r)$ has been deferred to this Appendix.

The first stage of the derivation involves differentiating eqn.(3.15) three times. Omitting the details, this process then yields the result

$$
\begin{gathered}
r t_{g}^{\prime \prime \prime}+3 t_{g}^{\prime \prime}=\frac{3}{4} \rho+\frac{9}{4} r \rho^{\prime}+\frac{9 r^{2}}{8} \rho^{\prime \prime}+\frac{r^{3}}{8} \rho^{\prime \prime \prime} \\
-n^{2} e^{-r^{2} / 2}\left[\frac{9 \pi^{3 / 2}}{2^{1 / 2}}+14 \pi\right]+n^{2} r^{2} e^{-r^{2} / 2}\left[\frac{33 \pi^{3 / 2}}{2^{1 / 2}}+16 \pi\right] \\
+12 \pi n^{2} e^{-r^{2}}-48 \pi n^{2} r^{2} e^{-r^{2}}+16 \pi n^{2} r^{4} e^{-r^{2}} \\
-n^{2} r^{4} e^{-r^{2} / 2}\left[\frac{13 \pi^{3 / 2}}{2^{1 / 2}}+2 \pi\right]+n^{2} r^{6} e^{-r^{2} / 2}\left[\frac{\pi^{3 / 2}}{2^{1 / 2}}\right]
\end{gathered}
$$

Next, we employ eqn.(4) of ref.10 to remove $\exp \left(-r^{2}\right)$ :

$$
2 \exp \left(-r^{2}\right)=\left\{c^{-1}\left[\left(1+r^{2}\right) \rho(r)+\left(r+r^{3}\right) \rho^{\prime}\right]-\frac{1}{4}\left(\frac{\pi}{2}\right)^{1 / 2}\left(7-4 r^{2}+r^{4}\right) \exp \left(-\frac{r^{2}}{2}\right)\right\}
$$

where

$$
c=2 \pi^{3 / 2} /\left[5 \pi^{1 / 2}+8\right]
$$

Thus, rewriting eqn.(A1) using eqn.(A2) leads to the form

$$
\begin{gathered}
r t_{g}^{\prime \prime \prime}+3 t_{g}^{\prime \prime}-\frac{3}{4} \rho+\frac{9}{4} r \rho^{\prime}+\frac{9 r^{2}}{8} \rho^{\prime \prime}+\frac{r^{3}}{8} \rho^{\prime \prime \prime} \\
-8 \pi n^{2} r^{4}\left[c^{-1}\left\{\left(1+r^{4}\right) \rho(r)+\left(r+r^{3}\right) \rho^{\prime}(r)\right\}\right] \\
+24 \pi n^{2} r^{2}\left[c^{-1}\left\{\left(1+r^{4}\right) \rho(r)+\left(r+r^{3}\right) \rho^{\prime}(r)\right\}-6 \pi n^{2} r^{2}\left(\frac{\pi}{2}\right)^{1 / 2}\left(7-4 r^{2}+r^{4}\right) \exp \left(-\frac{r^{2}}{2}\right)\right] \\
+2 \pi n^{2} r^{4}\left[\left(\frac{\pi}{2}\right)^{1 / 2}\left(7-4 r^{2}+r^{4}\right) \exp \left(-\frac{r^{2}}{2}\right)\right]+\frac{3 \pi}{2} n^{2}\left(\frac{\pi}{2}\right)\left(7-4 r^{2}+r^{4}\right) \exp -\frac{r^{2}}{2} \\
-n^{2} e^{-r^{2} / 2}\left[\frac{9 \pi^{3 / 2}}{2^{1 / 2}}+14 \pi\right]+n^{2} r^{2} e^{-r^{2} / 2}\left[\frac{33 \pi^{3 / 2}}{2^{1 / 2}}+6 \pi\right]
\end{gathered}
$$

or

$$
\begin{gathered}
r t_{g}^{\prime \prime \prime}+3 t_{g}^{\prime \prime}-\frac{3}{4} \rho+\frac{9}{4} r \rho^{\prime}+\frac{9 r^{2}}{8} \rho^{\prime \prime}+\frac{r^{3}}{8} \rho^{\prime \prime \prime} \\
+24 \pi n^{2} c^{-1} r^{2}\left[\left\{1+r^{4}\right\} \rho(r)+\left(r+r^{3}\right) \rho^{\prime}(r)\right]-8 \pi n^{2} c^{-1} r^{4}\left[\left\{1+r^{4}\right\} \rho(r)+\left(r_{r}^{3}\right) \rho^{\prime}(r)\right] \\
-6 \pi n^{2} c^{-1}\left[\left(1+r^{4}\right) \rho(r)+\left(r+r^{3}\right) \rho^{\prime}(r)\right]
\end{gathered}
$$




$$
\begin{gathered}
=-6 \pi\left(\frac{\pi}{2}\right)^{1 / 2} n^{2}\left[7 r^{2}-4 r^{4}+r^{6}\right] \exp \left(-\frac{r^{2}}{2}\right) \\
+2 \pi\left(\frac{\pi}{2}\right)^{1 / 2} n^{2}\left[7 r^{4}-4 r^{6}+r^{8}\right] \exp \left(-\frac{r^{2}}{2}\right) \\
+\frac{3 \pi}{2}\left(\frac{\pi}{2}\right)^{1 / 2} n^{2}\left[7-4 r^{2}+r^{4}\right] \exp \left(-\frac{r^{2}}{2}\right)
\end{gathered}
$$

The final step is to use eqn.(6) of ref.10 to eliminate $\exp \left(-\frac{r^{2}}{2}\right)$ from eqn.(A4), namely

$$
\left(\frac{\pi}{2}\right)^{1 / 2} \exp \left(-\frac{r^{2}}{2}\right)=\frac{4}{\left(r^{3}-r\right)} c^{-1}\left[\left(2 r+2 r^{3}\right) \rho(r)+\left(2+3 r^{2}\right) \rho^{\prime}+r \rho^{\prime \prime}\right]
$$

It is natural to multiply through by the factor $\left(r^{3}-r\right)$ appearing in the denominator of eqn.(A5) and then we obtain

$$
\begin{gathered}
\left(r^{4}-r^{2}\right) t_{g}^{\prime \prime \prime}+3\left(r^{3}-r\right) t_{g}^{\prime \prime}-\frac{3}{4}\left(r^{3}-r\right) \rho+\frac{9}{4}\left(r^{4}-r^{2}\right) \rho^{\prime} \\
+\frac{9}{8}\left(r^{5}-r^{3}\right) \rho^{\prime \prime}+\frac{\left(r^{6}-r^{4}\right)}{8} \rho^{\prime \prime \prime}+24 \pi n^{2} c^{-1}\left(r^{3}-r\right)\left(r^{2}+r^{6}\right) \rho(r) \\
+24 \pi n^{2} c^{-1}\left(r^{3}+r^{5}\right)\left(r^{3}-r\right) \rho^{\prime}-8 \pi n^{2} c^{-1}\left(r^{4}+r^{8}\right)\left(r^{3}-r\right) \rho(r) \\
-8 \pi n^{2} c^{-1}\left(r^{5}+r^{7}\right)\left(r^{3}-r\right) \rho^{\prime}-6 \pi n^{2} c^{-1}\left(1+r^{4}\right)\left(r^{3}-r\right) \rho \\
-6 \pi n^{2} c^{-1}\left(r^{3}-r\right)\left(r^{3}+r\right) \rho^{\prime} \\
=-6 \pi n^{2}\left(7 r^{2}-4 r^{4}+r^{6}\right) 4 c^{-1}\left[\left(2 r+2 r^{3}\right) \rho(r)+\left(2+3 r^{2}\right) \rho^{\prime}+r \rho^{\prime \prime}\right] \\
+2 \pi n^{2}\left(7 r^{4}-4 r^{6}+r^{8}\right) 4 c^{-1}\left[\left(2 r+2 r^{3}\right) \rho+\left(2+3 r^{2}\right) \rho^{\prime}+r \rho^{\prime}+r \rho^{\prime \prime}\right] \\
+\frac{3 \pi}{2} n^{2}\left(7-4 r^{2}+r^{4}\right) 4 c^{-1}\left[\left(2 r+2 r^{3}\right) \rho+\left(2+3 r^{2}\right) \rho^{\prime}+r \rho^{\prime \prime}\right] .
\end{gathered}
$$

Hence the results for $R_{i}(r)$ are found to be as in the following eqns.(A7)-(A10).

Since $n^{2} c^{-1} \pi=\left(8 \pi^{3}\right)^{-1}$ one finds first of all that

$$
R_{0}(r)=\left[-\frac{3}{4} r+\frac{3}{4} r^{3}\right]+\left(8 \pi^{3}\right)^{-1}\left[78 r-20 r^{3}+34 r^{5}+242 r^{7}-88 r^{9}+24 r^{11}\right]
$$

which is evidently an odd polynomial of $O\left(r^{11}\right)$.

Finding the coefficient of $\rho^{\prime}$ from eqn.(A6) yields the result

$$
R_{1}(r)=-\frac{9}{4} r^{2}+\frac{9}{4} r^{4}-\left(8 \pi^{3}\right)^{-1}\left[390 r^{2}+236 r^{4}-368 r^{6}+176 r^{8}-32 r^{10}\right]
$$

i.e. an even polynomial of $O\left(r^{10}\right)$.

Similarly $R_{2}(r)$ is found to be the $9^{\text {th }}$ order polynomial

$$
R_{2}(r)=-\frac{9}{8} r^{3}+\frac{9}{8} r^{5}-\left(8 \pi^{3}\right)^{-1}\left[42 r-192 r^{3}+158 r^{5}-56 r^{7}+8 r^{9}\right]
$$

and finally

$$
R_{3}(r)=-\frac{r^{4}}{8}+\frac{r^{6}}{8}
$$

This completes the result (3.16), giving the kinetic energy density $t_{g}(r)$ in terms of $\rho(r)$ and its low-order derivatives. 


\section{References}

1. K.A. Dawson and N.H. March, J. Chem. Phys. 81, 5850 (1984).

2. M. Moshinsky, Am. J. Phys. 36, 52 (1968).

3. For a summary, see L. Ballentine, Quantum Mechanics, Prentice-Hall, 1990.

4. N. Kestner and O. Sinanoglu, Phys. Rev. 128, 2687 (1962).

5. S. Kais, D.R. Herschbach and R.D. Levine, J. Chem. Phys. 91, 7791 (1989).

6. S. Kais, D.R. Herschbach, N.C. Handy, C.W. Murray and G.J. Laming, J. Chem. Phys. 99, 417 (1993).

7. N.H. March, I.A. Howard, I. Nagy and S. Rubio, Phys. Lett. A288, 101 (2001).

8. A. Holas and N.H. March, Phys. Rev. A51, 2040 (1995).

9. Z. Qian and V. Sahni, Phys. Rev. A57, 2527 (1998).

10. N.H. March, T. Gál and Á. Nagy, Chem. Phys. Lett. 292, 384 (1998).

11. See, for example, N.H. March, J. Math. Phys. 38, 2037 (1997).

12. C. Schwartz, Ann. Phys. NY 6, 156 (1959).

13. C.F. von Weizsäcker, Zeits für Physik, 96, 431 (1935).

14. T. Kato, Commun. Pure Appl. Math. 10, 151 (1957). 